\title{
Model For Bending Actuators That Use Electrostrictive Graft Elastomers
}

\author{
Robert C. Costen, Ji Su, and Joycelyn S. Harrison \\ Advanced Materials and Processing Branch \\ NASA Langley Research Center, Hampton, VA 23681
}

\begin{abstract}
Recently, it was reported that an electrostrictive graft elastomer exhibits large electric field-induced strain (4\%). Combined with its high mechanical modulus, the elastomer can offer very promising electromechanical properties, in terms of output mechanical energy density, for an electroactive polymeric material. Therefore, it has been considered as one of the candidates that can be used in high performance, low mass actuation devices in many aerospace applications. Various bilayer-based bending actuators have been designed and fabricated. An analytic model based on beam theory in the strength of materials has been derived for the transverse deflection, or curvature, and the longitudinal strain of the bi-layer beam. The curvature and strain are functions of the applied voltage and the thickness, width, and Young's modulus of the active and passive layers. The model can be used to optimize the performance of electrostrictive graft elastomer-based actuators to meet the requirements of various applications. In this presentation, optimization and sensitivity studies are applied to the bending performance of such actuators.
\end{abstract}

Keywords: Electrostrictive, electromechanical, graft elastomers, actuators, modeling.

\section{INTRODUCTION}

Materials that sustain mechanical displacement under controlled electrical excitation are needed as actuators for many applications. For aerospace applications, there is also a need for low mass, high performance, and ease of processability, which are inherent characteristics of electroactive materials. Electroactive polymeric elastomers that show electromechanical activity, especially large electric field induced strain, are being considered as potential candidates for such applications. Existing materials include polyurethane elastomers [1-3] and silicone rubber [4,5]. Recently, we have demonstrated an electrostrictive response in graft elastomers [6,7]. The elastomers offer large electric field induced strain and high mechanical modulus. Therefore, a high electromechanical output power, or high strain energy density is achieved. In addition to the high performance as a new class of electromechanically active polymeric materials, the electrostrictive graft elastomers also offer such advantages as excellent processability and electrical and mechanical toughness.

Smart materials, such as sensor and actuator materials, are finding many applications in space technologies. However, lightweight, low cost, and high performance are still challenges in the development of these types of functional materials. Recently, an electrostrictive graft elastomeric polymer was discovered at NASA Langley Research Center. It offers large electric field induced strain (4\%). This material can be used in many NASA space technologies that include position and tension control of functional membranes, aerodynamic and flow control of spacecraft, and development of self-adjustable multifunctional membranes as well as micro-electro-mechanical-systems (MEMS). Using the material, several prototype actuators have been developed. It was found that the performance of the actuators using the electrostrictive graft elastomers depends on the configuration, materials selection, and fabrication. This work is intended to help optimize the performance of the actuators by means of design and modeling.

\section{BASIC CONFIGURATION}

Figure 1 shows the basic configuration of a bending actuator that uses electrostrictive graft elastomers. The active layer was coated with gold electrodes on opposite sides of the graft elastomer film. The active layer was bonded to a passive graft elastomer layer that has no electrode coating. The adhesive was a thin epoxy layer. Figures $2 \mathrm{a}$ and $2 \mathrm{~b}$ show the fabricated electrostrictive graft elastomer-based bending actuator in unexcited and excited states, respectively. 


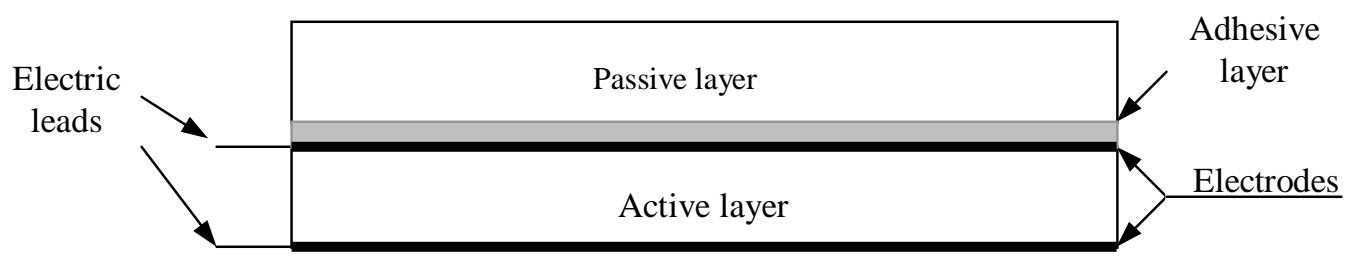

Figure 1: Basic configuration of a bending actuator using the electrostrictive graft elastomers.
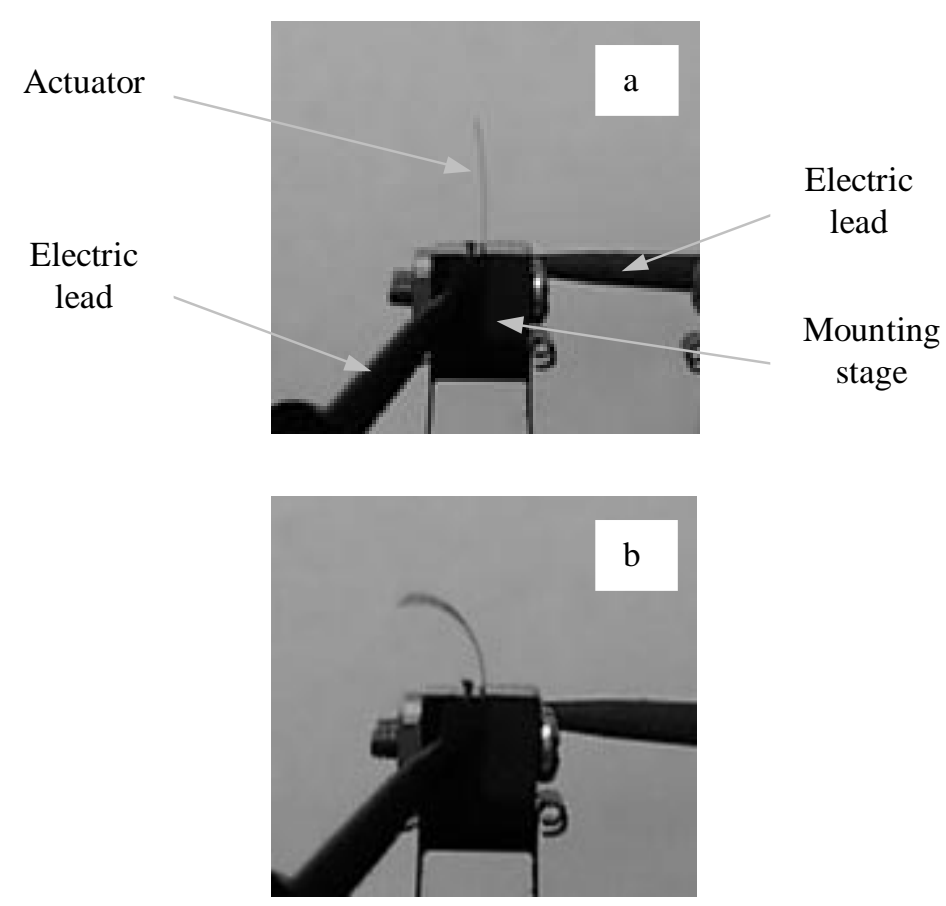

Figure 2: The bending actuator in the unexcited state (a) and the excited state (b).

\section{DERIVATION AND OPTIMIZATION OF MODEL}

\subsection{Derivation}

We shall attempt to develop an analytic model for the transverse curvature $\kappa$ and longitudinal strain $\varepsilon$ of a two- layer, unrestrained beam with one layer electrostrictive and the other passive. The model is based on beam theory in the strength of materials, as presented in [8]. Of the two layers comprising the beam, the electrostrictive layer is layer 1 and the passive layer is layer 2. The unexcited model beam is shown in Figure 3. In the unexcited state, the beam is flat and of length $L$. The height $h$, width $b$, and Young's modulus $E$ can be different for the two layers. Plane cross-sections of the beam are assumed to remain plane under flexure so that strains vary directly with their distance from the neutral axis, where the flexural strain is zero. Strains are assumed not to exceed the yield point. 


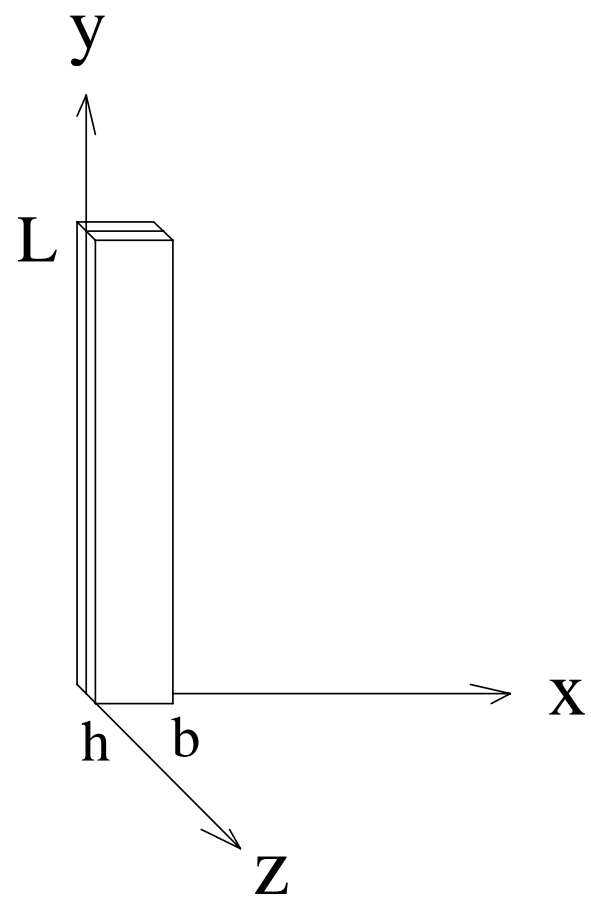

Figure 3: Model of a two-layer flat beam of length $L$, width $b$, and layer thickness $h$. The longitudinal direction lies along the $y$ - axis, and the transverse direction along the $z$ - axis.

For the laboratory prototype shown in Figure $2, L=0.035 \mathrm{~m}, h_{1}=h_{2}=21 \times 10^{-6} \mathrm{~m}$, and $b_{1}=b_{2}=5.75 \times 10^{-3} \mathrm{~m}$. The appropriate equations for developing this model are presented by F. L. Singer [8]. As in the treatment of thermal stresses, the electrostrictive layer is allowed to freely strain under excitation. To maintain longitudinal force equilibrium, the electrostrictive layer is then brought into compression and the passive layer into tension. The resulting longitudinal strain $\varepsilon$ of the beam is given by

$$
\varepsilon=\frac{e_{a}}{\left(\frac{E_{2} b_{2} h_{2}}{E_{1} b_{1} h_{1}}+1\right)}
$$

where $e_{a}$ is the free strain of the electrostrictive layer, as given by

$$
e_{a}=m\left(\frac{V_{1}}{h_{1}}\right)^{2}
$$

where $m=1.535 \times 10^{-18} \mathrm{~m}^{2}$ (volt) $)^{-2}$ is the electrostrictive constant for the graft elastomer, and $V_{1}$ is the voltage applied across the electrostrictive layer. With (1b) substituted for $e_{a}$, the longitudinal strain of the beam becomes

$$
\varepsilon=\frac{m V_{1}^{2}}{h_{1}^{2}\left(\frac{E_{2} b_{2} h_{2}}{E_{1} b_{1} h_{1}}+1\right)}
$$


The corresponding bending moment $M$ is given by

$$
M=\frac{\left(h_{1}+h_{2}\right) e_{a}}{2\left(\frac{1}{E_{1} b_{1} h_{1}}+\frac{1}{E_{2} b_{2} h_{2}}\right)}
$$

The beam curvature $\kappa=1 / \rho$, where $\rho$ is the radius of curvature that results from this moment, is given by

$$
\kappa=\frac{M}{E_{2} I}
$$

where $I$ is the moment of inertia about the neutral axis of the equivalent homogeneous beam having Young's modulus $E_{2}$ everywhere. In this equivalent homogeneous beam, width $b_{1}$ is replaced by its equivalent width $\left(b_{1} E_{1}\right) / E_{2}$. The neutral axis is the centroidal axis of the equivalent cross-section. The resultant expression for $I$ is given by

$$
I=\frac{\left(E_{1} b_{1}\right)^{2} h_{1}{ }^{4}+2 E_{1} b_{1} E_{2} b_{2} h_{1} h_{2}\left(2 h_{1}{ }^{2}+3 h_{1} h_{2}+2 h_{2}{ }^{2}\right)+\left(E_{2} b_{2}\right)^{2} h_{2}{ }^{4}}{12 E_{2}\left(E_{1} b_{1} h_{1}+E_{2} b_{2} h_{2}\right)}
$$

and the curvature $\kappa$ becomes

$$
\kappa=\frac{6 E_{1} b_{1} E_{2} b_{2} h_{1} h_{2}\left(h_{1}+h_{2}\right) e_{a}}{\left(E_{1} b_{1}\right)^{2} h_{1}^{4}+2 E_{1} b_{1} E_{2} b_{2} h_{1} h_{2}\left(2 h_{1}{ }^{2}+3 h_{1} h_{2}+2 h_{2}^{2}\right)+\left(E_{2} b_{2}\right)^{2} h_{2}^{4}}
$$

Substituting (1b) for $e_{a}$ and dividing the numerator and denominator by $E_{1} b_{1} E_{2} b_{2}$ give finally

$$
\kappa=\frac{6 h_{2}\left(h_{1}+h_{2}\right) m V_{1}^{2}}{h_{1}\left[\alpha h_{1}{ }^{4}+2 h_{1} h_{2}\left(2 h_{1}{ }^{2}+3 h_{1} h_{2}+2 h_{2}{ }^{2}\right)+\frac{h_{2}{ }^{4}}{\alpha}\right]}
$$

where

$$
\alpha=\frac{E_{1} b_{1}}{E_{2} b_{2}}
$$

This expression is a general formula for the unrestrained curvature of a two-layer beam with layer 1 electrostrictive and layer 2 passive.

\subsection{Optimizations}

Besides $m$ and $V_{1}$, the curvature $\kappa$ is a function of $h_{1}, h_{2}$, and $\alpha$. We can maximize the curvature with respect to $\alpha$ by taking $\partial \kappa / \partial \alpha=0$, which yields the $\alpha$-optimization condition

$$
\alpha=\left(\frac{h_{2}}{h_{1}}\right)^{2}
$$


When this condition is met, $\partial^{2} \kappa / \partial \alpha^{2}$ is negative, which confirms that $\kappa$ is a maximum with respect to $\alpha$. Therefore, for given values of $h_{1}$ and $h_{2}$, condition (6) gives the value of $\alpha$ that maximizes the curvature $\kappa$. Also, when (6) is satisfied, the curvature $\kappa$ is much less sensitive to small changes in $\alpha$ (small changes in $E_{1}, b_{1}, E_{2}$, or $b_{2}$ ) than to small changes in $h_{1}$ or $h_{2}$.

We can alternatively maximize the curvature $\kappa$ with respect to $h_{2}$ by taking $\partial \kappa / \partial h_{2}=0$, which yields the $h_{2}$ optimization condition

$$
2 h_{2}{ }^{3}+3 h_{2}{ }^{2} h_{1}-\alpha h_{1}^{3}=0
$$

This cubic equation has only one root for $h_{2}$ that is real and positive for practical values of $\alpha$

$$
h_{2}=f(\alpha) h_{1}
$$

where

$$
f(\alpha)=\frac{1}{2}\left\{\left[-1+2 \alpha+2\left(\alpha^{2}-\alpha\right)^{\frac{1}{2}}\right]^{\frac{1}{3}}+\frac{1}{\left[-1+2 \alpha+2\left(\alpha^{2}-\alpha\right)^{\frac{1}{2}}\right]^{\frac{1}{3}}}-1\right\}
$$

A plot of $f(\alpha)$ versus $\alpha$ is shown in Figure 4 .

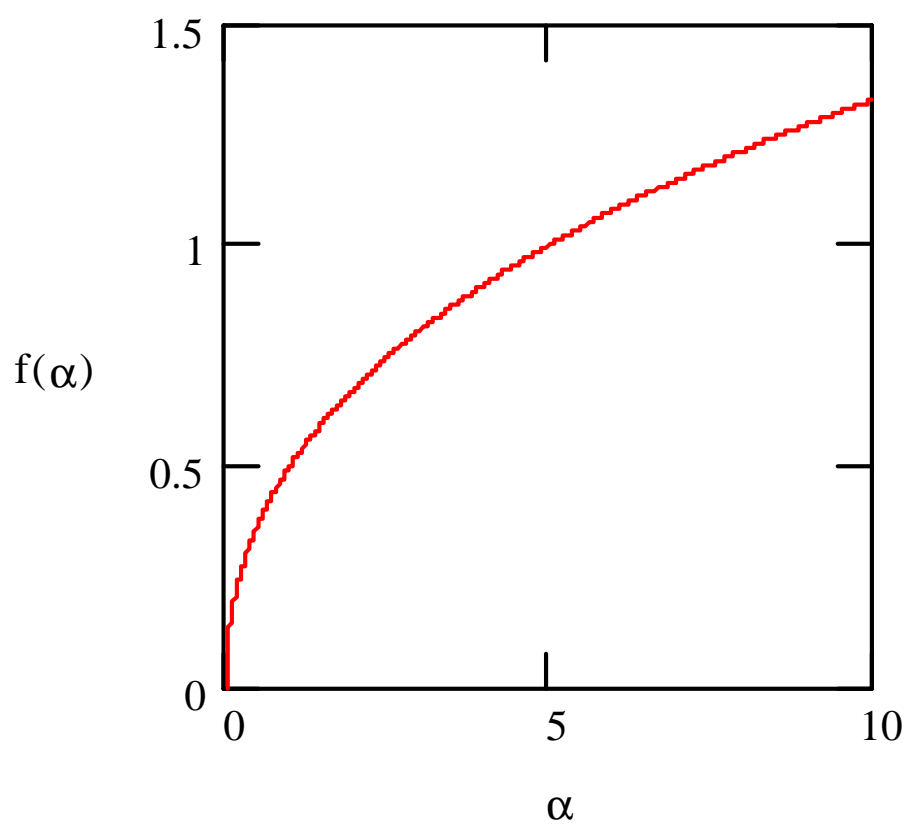

Figure 4: Plot of $f(\alpha)$ versus $\alpha$, where $f(\alpha)$ is given by (8b) and $\alpha$ by (5c).

Thus, for any given values of $h_{1}$ and $\alpha$, the value of $h_{2}$ that maximizes the curvature $\kappa$ is given by (8a). 
If we attempt to maximize the curvature $\kappa$ with respect to $h_{1}$ by taking $\partial \kappa / \partial h_{1}=0$, we find that there are no positive real solutions for $h_{1}$. Therefore, for given values of $h_{2}$ and $\alpha$, there is no value for $h_{1}$ that maximizes $\kappa$. The reason that no optimum value for $h_{1}$ exists is clarified by plotting contours of $\ln (\kappa)$ on the $h_{1}-h_{2}$ plane, as shown in Figure 5.

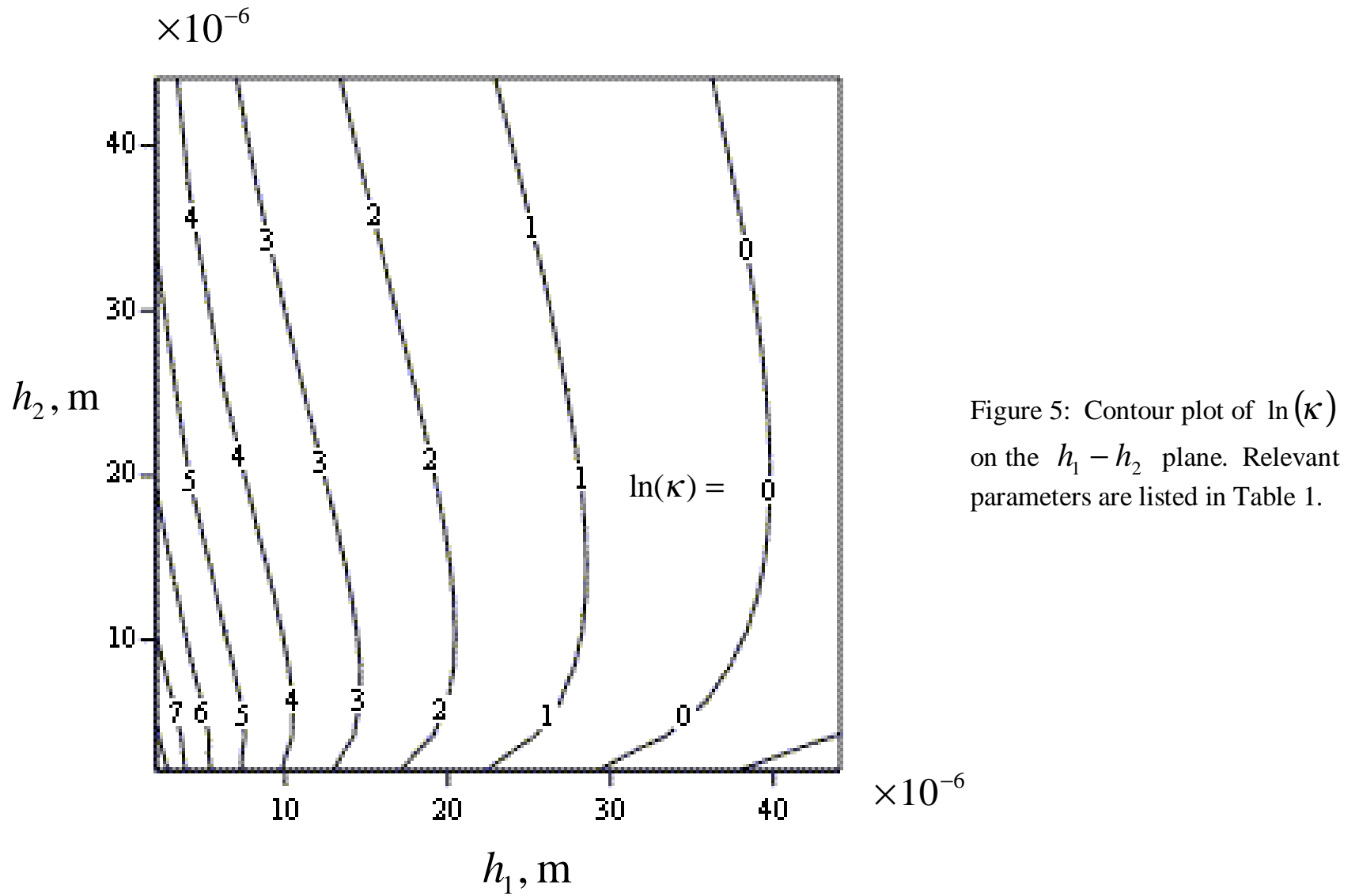

As $h_{2}$ increases along a vertical line, $\kappa$ increases until $h_{2}$ reaches the value given by (8a), after which $\kappa$ decreases. In contrast, as $h_{1}$ decreases along a horizontal line, $\kappa$ continues to increase monotonically without leveling off to a maximum value. Therefore, for given values of $h_{1}$ and $\alpha$, there exists a value of $h_{2}$ that maximizes $\kappa$; but for given values of $h_{2}$ and $\alpha$, one can always increase $\kappa$ further by decreasing $h_{1}$.

\section{SENSITIVITY STUDIES}

At this point the model is not sufficiently close to the laboratory prototype to make a quantitative comparison. The laboratory prototype actually contains five layers, as shown in Figure 1. Besides the electroactive graft elastomer layer and the passive graft elastomer layer of the model, there is the adhesive layer between them and two metallic gold layers that coat the electroactive layer and across which the voltage is applied. The gold layers reduce the effective longitudinal strain of the electroactive layer and increase the beam's resistance to bending. The adhesive layer also has similar effects. Eventually we hope to bring the model and prototype into agreement. At present, we will only attempt to gain qualitative insights from the model by performing sensitivity studies. 
The sensitivity studies apply to a simplified version of the laboratory prototype where the effects on curvature of the two gold layers and the adhesive layer are neglected. The first study starts from a baseline state (indicated by the superscript (0)) that is $\alpha$ - optimized by satisfying (6). The applied voltage $V_{1}^{0}=215$ volts, which gives a curvature to the beam of $\kappa^{0}=5.75 \mathrm{~m}^{-1}$ and radius of curvature $\rho^{0}=0.174 \mathrm{~m}$. The other baseline parameters for sensitivity study 1 are given in Table 1.

Table 1. $\alpha$-Optimized Baseline State For Sensitivity Study 1

$$
\begin{aligned}
& E_{1}{ }^{0}=E_{2}{ }^{0}=580 \times 10^{6} \mathrm{~Pa} \\
& b_{1}{ }^{0}=b_{2}{ }^{0}=5.75 \times 10^{-3} \mathrm{~m} \\
& h_{1}{ }^{0}=h_{2}{ }^{0}=21 \times 10^{-6} \mathrm{~m} \\
& I^{0}=3.55 \times 10^{-17} \mathrm{~m}^{4} \\
& m^{0}=1.535 \times 10^{-18} \mathrm{~m}^{2}(\text { volts })^{-2} \\
& V_{1}^{0}=215 \text { volts } \\
& e_{a}{ }^{0}=1.61 \times 10^{-4} \\
& M^{0}=1.18 \times 10^{-7} \mathrm{~N} \mathrm{~m} \\
& \kappa^{0}=5.75 \mathrm{~m}^{-1} \\
& \rho^{0}=0.174 \mathrm{~m} \\
& \varepsilon^{0}=8.04 \times 10^{-5} \\
& L^{0}=0.035003 \mathrm{~m}
\end{aligned}
$$

Because the baseline longitudinal strain $\varepsilon^{0}$ due to the baseline voltage $V_{1}^{0}$, as given in Table 1, is so small, the increase in the beam length $L^{0}$ is negligible, and it remains so throughout both sensitivity studies.

In each sensitivity study, various design parameters are varied one at a time, and we calculate the effects on the unrestrained curvature $\kappa(5 b)$ and longitudinal strain $\varepsilon(1 \mathrm{c})$, as shown in Table 2.

Table 2. Sensitivity Study 1 For $\alpha$-Optimized Baseline State

\begin{tabular}{l|l|l|l|l|l}
\cline { 2 - 6 } & $e_{a} / e_{a}{ }^{0}$ & $M / M^{0}$ & $I / I^{0}$ & $\kappa / \kappa^{0}$ & $\varepsilon / \varepsilon^{0}$ \\
\hline$m / m^{0}=1.1$ & 1.1 & 1.1 & 1 & 1.1 & 1.1 \\
\hline$V_{1} / V_{1}^{0}=1.1$ & 1.21 & 1.21 & 1 & 1.21 & 1.21 \\
\hline$h_{1} / h_{1}^{0}=.9$ & 1.23 & 1.11 & .86 & 1.30 & 1.17 \\
\hline$h_{2} / h_{2}{ }^{0}=.9$ & 1 & .9 & .86 & 1.05 & 1.05 \\
\hline$E_{1} / E_{1}^{0}=1.1$ & 1 & 1.05 & 1.05 & 1 & 1.05 \\
\hline$E_{2} / E_{2}^{0}=.9$ & 1 & .95 & 1.05 & 1 & 1.05 \\
\hline$b_{1} / b_{1}{ }^{0}=1.1$ & 1 & 1.05 & 1.05 & 1 & 1.05 \\
\hline$b_{2} / b_{2}{ }^{0}=.9$ & 1 & .95 & .95 & 1 & 1.05 \\
\hline & & & &
\end{tabular}


From this table, we see that a 10 percent increase in the electrostrictive constant $m$ results in a 10 percent increase in the curvature $\kappa$ and a 10 percent increase in the longitudinal strain $\varepsilon$ of the beam. The increase in $\kappa$ results from a 10 percent increase in the bending moment $M$ due to a 10 percent increase in the free strain $e_{a}$ of the active layer. This increase in $e_{a}$ also accounts for the increase in $\varepsilon$. In the formulas for $\kappa$ and $\varepsilon$, the voltage $V_{1}$ enters as a squared quantity. Therefore, a 10 percent increase in $V_{1}$ results in a 21 percent increase in the curvature $\kappa$ and a 21 percent increase in the longitudinal strain $\varepsilon$.

A 10 percent decrease in the thickness of the active layer $h_{1}$ gives a 30 percent increase in the curvature $\kappa$ and a 17 percent increase in $\varepsilon$. In this case, the increase in $\kappa$ results from an 11 percent increase in the bending moment $M$ and a 14 percent decrease in the moment of inertia $I$. A 10 percent decrease in the thickness of the passive layer $h_{2}$ gives a 5 percent increase in $\kappa$ and a 5 percent increase in $\varepsilon$. These changes result from a 10 percent decrease in $M$ and a 14 percent decrease in $I$. We infer that the curvature $\kappa$ of a beam could be tailored efficiently by varying the thickness of the active layer $h_{1}$ along its length. However, for the $\alpha$ - optimization to remain valid for large thickness changes, both layers may need to be tailored.

Ten percent changes in Young's moduli $E_{1}$ and $E_{2}$ or in the widths $b_{1}$ and $b_{2}$ can alter the longitudinal strain $\varepsilon$ by 5 percent but have a negligible effect on the curvature $\kappa$. The insensitivity of $\kappa$ to these changes confirms that $\partial \kappa / \partial \alpha=0$ when the $\alpha$ - optimization condition (6) is satisfied.

The second sensitivity study starts from a baseline state that is $h_{2}$ - optimized. According to Figure 4 , when $\alpha=1$, which corresponds to the laboratory prototype, $f(\alpha)=1 / 2$ and $h_{2}=h_{1} / 2$. This value for $h_{2}$ deviates from the laboratory prototype and from sensitivity study 1 . The baseline state for the second sensitivity study is given in Table 3 , where values that differ from the first study are indicated by arrows $(\rightarrow)$. In particular, the baseline curvature $\kappa^{0}$ is substantially greater in Table 3 than in Table 1.

Table 3. $h_{2}$-Optimized Baseline State For Sensitivity Study 2

$$
\begin{aligned}
& E_{1}{ }^{0}=E_{2}{ }^{0}=580 \times 10^{6} \mathrm{~Pa} \\
& b_{1}{ }^{0}=b_{2}{ }^{0}=5.75 \times 10^{-3} \mathrm{~m} \\
& h_{1}^{0}=21 \times 10^{-6} \mathrm{~m} \\
& \rightarrow h_{2}{ }^{0}=10.5 \times 10^{-6} \mathrm{~m} \\
& \rightarrow I^{0}=1.5 \times 10^{-17} \mathrm{~m}^{4} \\
& m^{0}=1.535 \times 10^{-18} \mathrm{~m}^{2} \text { (volts) }{ }^{-2} \\
& V_{1}^{0}=215 \text { volts } \\
& e_{a}{ }^{0}=1.61 \times 10^{-4} \\
& \rightarrow M^{0}=5.92 \times 10^{-8} \mathrm{~N} \mathrm{~m} \\
& \rightarrow \kappa^{0}=6.81 \mathrm{~m}^{-1} \\
& \rightarrow \rho^{0}=0.147 \mathrm{~m} \\
& \rightarrow \varepsilon^{0}=1.07 \times 10^{-4} \\
& \rightarrow L^{0}=0.035004 \mathrm{~m}
\end{aligned}
$$


As before, the baseline values are varied one at a time by 10 percent, and the effects on curvature $\kappa$, longitudinal strain $\varepsilon$, and other relevant quantities are given in Table 4.

Table 4. Sensitivity Study 2 For $h_{2}$-Optimized Baseline State

\begin{tabular}{l|l|l|l|l|l}
\cline { 2 - 6 } & $e_{a} / e_{a}^{0}$ & $M / M^{0}$ & $I / I^{0}$ & $\kappa / \kappa^{0}$ & $\varepsilon / \varepsilon^{0}$ \\
\hline$m / m^{0}=1.1$ & 1.1 & 1.1 & 1 & 1.1 & 1.1 \\
\hline$V_{1} / V_{1}^{0}=1.1$ & 1.21 & 1.21 & 1 & 1.21 & 1.21 \\
\hline$h_{1} / h_{1}^{0}=.9$ & 1.23 & 1.11 & .81 & 1.37 & 1.19 \\
\hline$h_{2} / h_{2}^{0}=.9$ & 1 & .9 & .9 & 1 & 1.03 \\
\hline$E_{1} / E_{1}^{0}=.9$ & 1 & .96 & .95 & 1.02 & .96 \\
\hline$E_{2} / E_{2}^{0}=1.1$ & 1 & 1.06 & .95 & 1.02 & .97 \\
\hline$b_{1} / b_{1}{ }^{0}=.9$ & 1 & .96 & .95 & 1.02 & .96 \\
\hline$b_{2} / b_{2}{ }^{0}=1.1$ & 1 & 1.06 & 1.05 & 1.02 & .97 \\
\hline
\end{tabular}

A 10 percent decrease in $h_{1}$ gives a 37 percent increase in $\kappa$. Therefore, besides greater baseline curvature, the $h_{2}$ optimized state gives greater curvature sensitivity than the $\alpha$-optimized state. This result could change for different voltages, etc., and both optimizations should be considered in the design process. As expected for the $h_{2}$-optimization, the curvature $\kappa$ is insensitive to small changes in $h_{2}$; however, $\kappa$ now has gained some sensitivity to $E_{1}, b_{1}, E_{2}$, and $b_{2}$, which was missing in the $\alpha$-optimization.

\section{CONCLUDING REMARKS}

Beam theory in the strength of materials was used to obtain an analytic model for the transverse curvature and longitudinal strain of a two-layer actuator consisting of one electrostrictive layer and one passive layer. The layers can have different thicknesses, widths and Young's moduli. The curvature formula was optimized in two ways: (1) by varying the thickness of the passive layer and (2) by varying the ratio of the product of width and Young's modulus for both layers. The latter optimization was satisfied by a simplified version of the laboratory prototype. The former optimization required halving the thickness of the prototype's passive layer. Two sensitivity studies (one for each optimization) were performed with the model. Both studies showed that a 10 percent increase in the voltage across the electrostrictive layer gives a 21 percent increase in the curvature. A 10 percent decrease in the thickness of the electrostrictive layer gives a 30 percent increase in curvature for the optimization satisfied by the laboratory prototype and a 37 percent increase for the other optimization. The curvature is much less sensitive to small variations in the thickness of the passive layer. Therefore, the curvature of an actuator beam can be tailored efficiently along its length by varying the thickness of its active layer. However, for either optimization to remain valid for large thickness changes, both layers may need to be varied.

\section{REFERENCES}

1. M. Zhenyi, J. I. Scheinbeim, J. W. Lee, and B. A. Newman, J. Polym. Sci., Part B: Polym. Phys., 32, 2721 (1994).

2. Q. M. Zhang, J. Su, C. H. Kim, R. Ting, and R. Capps, J. Appl. Phys., 81, 2770 (1997).

3. J. Su, Q. M. Zhang, C. H. Kim, R. Y. Ting, and R. Capps, J. Appl. Polym. Sci., 65, 1363 (1997).

4. R. Pelrine, R. Kornbluh, and J. Joseph, Sensor and Actuators A: Physical, 64, 77 (1998).

5. R. Kornbluh, R. Pelrine, J. Joseph, R. Heydt, Q. Pei, and S. Chiba, Proceedings of SPIE, 3669, 149 (1999).

6. J. Su, J. S. Harrison, T. St. Clair, Y. Bar-Cohen, and S. Leary, Proceedings of MRS Fall Meeting, 600, 131, (1999).

7. J. Su, J. S. Harrison, and T. St. Clair, Proceedings of IEEE International Symposium on Application of Ferroelectrics, (2001).

8. F. L. Singer, Strength of Materials, Harper \& Brothers (1951). 